\title{
NeuroRegulation
}

\section{Editorial - Volume 2, Number 3}

Citation: Cannon, R. L. (2015). Editorial - Volume 2, Number 3. NeuroRegulation, 2(3), 110. http://dx.doi.org/10.15540/nr.2.3.110

Copyright: ( $)$ 2015. Cannon. This is an Open Access article distributed under the terms of the Creative Commons Attribution License (CC-BY).
*Address correspondence to: Rex L. Cannon, PhD, BCN, Neural Potential, 683 Edgebrook Ln., West Palm Beach, FL 33411, USA. Email: rexcannon@neuralpotential.com
Welcome to NeuroRegulation Volume 2, Number 3. In this issue we have several research papers and a review of quantitative electroencephalogram (qEEG) guided neurofeedback. Dr. Svetlana Malyutina and Dr. Dirk-Bart den Ouden examine the effects of transcranial direct current stimulation (tCDS) in aphasic disturbances and conclude this modality may prove useful in language dysfunctions. Dr. Jon Frederick, Kelli Dunn, and Dr. Thomas Collura provide pilot data on the relationship between EEG state discrimination and neurofeedback procedures and potential corresponding mechanisms inherent to these two processes. Stephanie Dreis, Angela Gouger, Edward Perez, Michael Russo, Michael Fitzsimmons, and Dr. Mark Jones provide pilot data examining the impact of using individualized qEEGguided protocols to reduce symptoms of anxiety. The authors discuss the findings and implications for further study. Finally, Dr. Nancy Wigton and Dr. Genomary Krigbaum provide a review of qEEGguided neurofeedback data. The authors examine trends and directions needed for future study. We thank all authors for their contribution to the scientific advancement of neurofeedback.
It is nearly time for our International Society for Neurofeedback and Research (ISNR) 23rd annual conference in Denver, Colorado. We would like to encourage all members and presenters to submit their research to NeuroRegulation. There are usually quite a large number of exceptional studies and theoretical constructs presented at our conference and we welcome all topics for NeuroRegulation. We would also like to encourage students to submit their research as well. We look forward to seeing you all in Denver.

Rex L. Cannon, PhD, BCN

Editor-in-Chief

Email: rexcannon@gmail.com

Published: October 8, 2015 